\title{
Recessive mutation in GALNT3 causes hyperphosphatemic familial tumoral calcinosis associated with chronic recurrent multifocal osteomyelitis
}

\author{
Jumana Albaramki ${ }^{1}$, Haitham Dmour ${ }^{1}$, Mohammad Shboul'2, Carine Bonnard ${ }^{2}$, \\ Byrappa Venkatesh ${ }^{3}$, Rasha Odeh ${ }^{1}$ \\ 1 Department of Pediatric, School of Medicine, The University of Jordan, Jordan, Amman; 2Institute of Medical Biology \\ and 3Institute of Molecular and Cell Biology, A*STAR, Singapore. E-mail: jumanabaramki@hotmail.com \\ Received: 25th October 2017, Revised: 29th December 2017, Accepted: 3nd January 2018
}

\begin{abstract}
SUMMARY: Albaramki J, Dmour H, Shboul M, Bonnard C, Venkatesh B, Odeh R. Recessive mutation in GALNT3 causes hyperphosphatemic familial tumoral calcinosis associated with chronic recurrent multifocal osteomyelitis. Turk J Pediatr 2019; 61: 130-133.

Hyperphosphatemic familial tumoral calcinosis is a rare autosomal recessive disorder that is characterized by persistent hyperphosphatemia and extra-articular calcifications. Three cases were previously reported with hyperphosphatemic familial tumoral calcinosis that were associated with chronic recurrent multifocal osteomyelitis, an autoinflammatory disorder that is characterized by recurrent episodes of bone pain. We describe here an 11-year-old child who was diagnosed with these two conditions and was found to carry a splice site mutation c.1524+1G>A in the GALNT3 gene.
\end{abstract}

Key words: calcinosis, osteomyelitis, hyperphosphatemia.

Hyperphosphatemic familial tumoral calcinosis (HFTC) is a rare autosomal recessive disorder (MIM 211900) that is characterized by enhanced renal tubular reabsorption of phosphate, hyperphosphatemia and presence of subcutaneous soft tissue periarticular calcifications around large joints. Mutations in three genes, FGF23 encoding the phosphaturic hormone FGF-23, GALNT3 that encodes UDP$\mathrm{N}$-acetyly-alpha-D-galactosamine polypepetide $\mathrm{N}$-acetylgalactosamine transferase 3 and $K L$ encoding alpha Klotho were identified as causative of HFTC.1 The literature contains only three reports that associate HFTC2-4 with chronic recurrent multifocal osteomyelitis (CRMO), an autoinflammatory disorder characterized by bone pain and fever with an unpredictable course of exacerbations and spontaneous remissions.5 We report an 11-year-old boy with CRMO and HFTC who carries a germ line homozygous mutation (c.1524+1G>A; p.K465_Y508del) in GALNT3 gene and analyze the current literature for this association.

\section{Case Report}

A Jordanian patient presented to the clinic at the age of 8 years with left leg pain, swelling and redness along with fever of five day duration, high markers of inflammation, as well as a firm non-tender swelling over the right elbow. Lower limb MRI showed abnormal marrow signal involving left tibial diaphysis with laminated periosteal reaction and surrounding soft tissue edema suggestive of an inflammatory process. Assuming bacterial osteomyelitis, he was treated with intravenous antibiotics for four weeks. Bone scan showed increased activity at mid left radius, mid right femur, mid left tibia and smaller foci of abnormal tracer in right mid fibula. Elbow $\mathrm{X}$-ray showed a calcified extra-articular lesion. $\mathrm{He}$ had a history of severe right leg pain and fever at the age of 3 years that was treated as bacterial osteomyelitis with antibiotics and drainage without bacterial growth on culture. He had also developed right elbow swelling that was thought to be an ossified hematoma and had repeated attacks of pain in both legs since age of three. Based on his clinical 


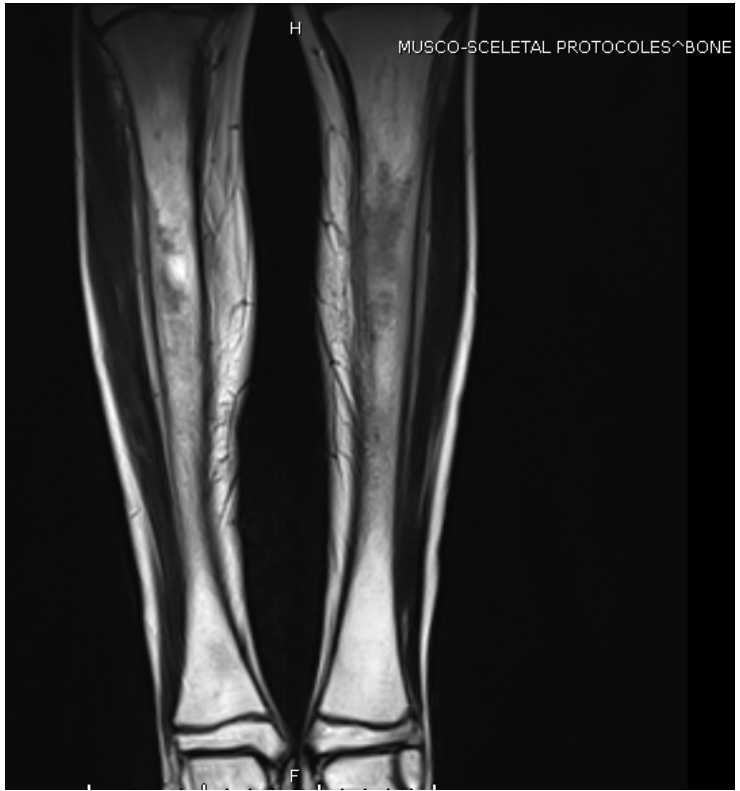

Fig. 1. Abnormal bilateral enhancement of the bone marrow involving the shaft of the tibia suggestive of an inflammatory process.

course of recurrent episodes of leg pain he was diagnosed with chronic relapsing multifocal osteomyelitis and was started on ibuprofen and steroids that was tapered over 9 months. His pain was partly controlled with steroids. He was then admitted to the hospital at the age of 10 years with pain in his hands, arms and lower limbs and the bone scan showed increased uptake in mandible, upper thoracic spine, left proximal forearm, right tibia, and right calcaneus suggestive of CRMO.

His clinically normal parents were first cousins, and his siblings were unaffected. During his last admission to the hospital he was found to have high serum phosphate $(8.7 \mathrm{mg} / \mathrm{dl})$, with normal calcium, vitamin $\mathrm{D}$, and parathyroid hormone. Tubular reabsorption of phosphate (TRP) was $98 \%$ despite the hyperphosphatemia. The diagnosis of HFTC was also considered due to the large elbow calcification. He was then placed on a low phosphate diet and calcium carbonate with poor compliance. A few months later he developed a round nodule over the right foot which was excised and found to be idiopathic calcinosis. Due to the fluctuation of the serum phosphate, acetazolamide was added at a dose of $250 \mathrm{mg}$ twice daily with a favorable response. He had another episode of CRMO involving both legs that was managed
A.

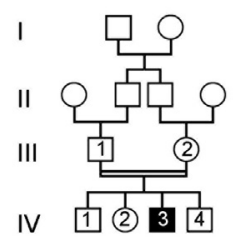

B.



Fig. 2. (A) Pedigree of the family. Closed symbol: affected individual, open symbols: unaffected individuals, double horizontal line: consanguineous marriage. (B) Electropherograms of the GALNT3 mutation obtained by Sanger sequencing. Parents (III.1 and III.2) are heterozygous/unaffected carriers; the affected individual is homozygous mutant for c. $1524+1 \mathrm{G}>\mathrm{A}$ mutation. The asterisk indicates the position of the mutation.

with naproxen. (Fig. 1).

Whole exome sequencing of the proband together with both parents identified a deleterious splice-site mutation (c.1524+1G $>$ A) in intron 8 of GALNT3 that causes mRNA mis-splicing

followed by non-sense mediated decay. With informed consent of the patient and family members, whole exome sequencing analyses were applied.

Segregation analysis was done to validate the splice site mutation as the cause for the disease. We confirmed that both parents were heterozygous and the affected child was homozygous for the mutant allele (Fig. 2).

\section{Discussion}

CRMO, an inherited autoinflammatory bone disease, is characterized by recurrent episodes of leg pain and swelling that mimics infectious osteomyelitis but with negative cultures. It has been associated with other inflammatory co-morbidities including skin inflammation. 5 CRMO has been also associated with tumoral calcinosis in children of Middle Eastern ethnicity2,3 however, genetic testing was not performed. Another case report4 described an African boy with an initial diagnosis of CRMO who later developed HTFC and for whom a nonsense mutation in GALNT, (c. $484 \mathrm{C}>\mathrm{T}$; p.R162X) was identified. Another child initially diagnosed with CRMO, who later developed normophostatemic tumoral calcinosis was also reported without genetic etiology description. 6 Based on these different reports, there seems to 
be an increased predisposition in children with CRMO to develop tumoral calcinosis whether with normal or high phosphate levels. Due to the limited number of cases associating these two conditions, it is unclear whether the child with CRMO has an increased predisposition to developing HFTC.

Mutations in GALNT3 have also been associated with hyperphosphatemic hyperosteosis syndrome (HHS) which is manifested by episodic leg pain and swelling, cortical hyperostosis and sclerosis, and periosteal reaction, thus overlapping with CRMO. Coexistence of HHS and HFTC conditions have been described before in the same patients7-9 with suggestion that the manifestations are alternatives to the same condition. Whether the clinical and radiological findings of these children with HHS may represent a continuum or a spectrum of CRMO is to be speculated.

The mutation c. $1524+1 \mathrm{G}>\mathrm{A}$ in GALNT3, identified in our case has been previously described causing HFTC in a Druze family10 with tumoral calcinosis and in ArabMoslem families with HFTC and HHS.9 The identification of c. $1524+1 \mathrm{G}>\mathrm{A}$ mutation in a Jordanian patient and other Arab-Moslem and Druze families supports the existence of a founder effect and suggests a common origin of this mutation in the middle east. Moreover, the phenotypic differences in all families, for example CRMO was not diagnosed in all these families, suggests a possible contribution of genetic modifiers that could modulate the disease phenotypes.

Few data are available regarding the optimal therapy for HFTC, such as low phosphate diet, or the use of phosphate binders such as calcium carbonate and sevelamer. ${ }^{11}$ Other treatments which include bisphosphonates ${ }^{12}$, acetazolamide $^{11,13}$ have variable success in controlling disease activity. In a recent case report, acetazolamide did not reduce phosphate levels, but increased calcium phosphate complex solubility by creating a state of metabolic acidosis. ${ }^{13}$

In conclusion there seems to be a genotypic and phenotypic association of CRMO and familial tumoral calcinosis that requires deeper exploration to identify the common pathophysiology. This report highlights the importance of following phosphate levels in children presenting with CRMO to attempt early treatment and avoid bone calcification.

\section{Acknowledgement}

This work was funded by a Strategic Positioning Fund on Genetic Orphan Diseases from the Agency for Science, Technology and Research (A*STAR) in Singapor.

\section{REFERENCES}

1. Farrow EG, Imel EA, White KE. Miscellaneous non-inflammatory musculoskeletal conditions. Hyperphosphatemic familial tumoral calcinosis (FGF23, GALNT3 and $\alpha$ Klotho). Best Pract Res Clin Rheumatol 2011; 25: 735-747.

2. Majeed SA. Chronic recurrent multifocal osteomyelitis associated with tumoral calcinosis. J Bone Joint Surg Br 1994; 76: 325-327.

3. Maus U, Ihme N, Schroeder S, et al. Chronic recurrent multifocal osteomyelitis and tumoral calcinosis--is there an association? Klin Padiatr 2007; 219: 277-281.

4. Demellawy DE, Chang N, de Nanassy J, Nasr A. GALNT3 gene mutation-associated chronic recurrent multifocal osteomyelitis and familial hyperphosphatemic familial tumoral calcinosis. Scand J Rheumatol 2015; 44: $170-172$.

5. Ferguson PJ, El-Shanti HI. Autoinflammatory bone disorders. Curr Opin Rheumatol 2007; 19: 492-498.

6. Yüksel HY, Yılmaz S, Gürbüzel M. Normophosphatemic type tumoral calcinosis associated with chronic recurrent multifocal osteomyelitis: A case report. Acta Orthop Traumatol Turc 2011;45:124-129.

7. Rafaelsen S, Johansson S, Ræder H, Bjerknes R. Longterm clinical outcome and phenotypic variability in hyperphosphatemic familial tumoral calcinosis and hyperphosphatemic hyperostosis syndrome caused by a novel GALNT3 mutation; case report and review of the literature. BMC Genet 2014; 24:15-98.

8. Ichikawa S, Baujat $\mathrm{G}$, Seyahi A, et al. Clinical variability of familial tumoral calcinosis caused by novel GALNT3 mutations. Am J Med Genet A 2010; 152A: 896-903.

9. Frishberg Y, Topaz O, Bergman R, et al. Identification of a recurrent mutation in GALNT3 demonstrates that hyperostosis-hyperphosphatemia syndrome and familial tumoral calcinosis are allelic disorders. J Mol Med (Berl) 2005; 83: 33-38.

10. Topaz O, Shurman DL, Bergman R, et al. Mutations in GALNT3, encoding a protein involved in O-linked glycosylation, cause familial tumoral calcinosis. Nat Genet 2004; 36:579-581.

11. Lammoglia JJ, Mericq V. Familial tumoral calcinosis caused by a novel FGF23 mutation: Response to induction of tubular renal acidosis with acetazolamide and the non-calcium phosphate binder sevelamer. Horm Res 2009; 71: 178-184. 
12. Balachandran K, Kamalanathan S, Sahoo JP, Das AK, Halanaik D. Differential response of idiopathic sporadic tumoral calcinosis to bisphosphonates. Indian J Endocrinol Metab 2014; 18: 521-525.
13. Finer G, Price HE, Shore RM, White KE, Langman CB. Hyperphosphatemic familial tumoral calcinosis: response to acetazolamide and postulated mechanisms. Am J Med Genet A 2014; 164A: 1545-1549. 